\title{
Probing the Turbulence Dissipation Range and Magnetic Field Strengths in Molecular Clouds
}

\author{
Hua-bai Li \\ Harvard-Smithsonian Center for Astrophysics, 60 Garden Street, Cambridge, MA 02138, USA \\ hli@cfa.harvard.edu \\ Martin Houde \\ Department of Physics and Astronomy, The University of Western Ontario, London, Ontario, \\ Canada N6A $3 K^{7}$ \\ houde@astro.uwo.ca
}

\begin{abstract}
We study the turbulent velocity dispersion spectra of the coexistent $\mathrm{HCN}$ and $\mathrm{HCO}^{+}$ molecular species as a function of length scale in the M17 star-forming molecular cloud. We show that the observed downward shift of the ion's spectrum relative to that of the neutral is readily explained by the existence of an ambipolar diffusion range within which ion and neutral turbulent energies dissipate differently. We use these observations to evaluate this decoupling scale and show how to estimate the strength of the plane-ofthe-sky component of the embedded magnetic field in a completely novel way.
\end{abstract}

Subject headings: ISM: clouds — ISM: individual (M17) — ISM: magnetic fields — Physical Data and Processes: turbulence

\section{Introduction}

Star formation is one of the very basic topics of astronomy. It was proposed as early as in the 18th century, by Immanuel Kant and Simon de Laplace independently, that stars originate from clouds of matter contracting under their own gravitation. This point of view is supported by modern observations, which show a high correlation between the positions of newly formed stars and those of molecular clouds. Molecular clouds are made up primarily of molecular hydrogen with a mean number density of approximately $10^{2} \mathrm{~cm}^{-3}$. Some of the clouds are as big as $10-100 \mathrm{pc}$ with masses reaching $10^{4}-10^{6} M_{\odot}$, while the typical temperature of an individual cloud is only $10-50 \mathrm{~K}$. Since the Jeans mass, which is the minimum cloud mass needed to overcome thermal motion and bring gravitational collapse, is approximately $20 M_{\odot}$, it follows that molecular clouds are highly gravitationally bound. On the other hand, the star formation efficiency, defined as the 
ratio of stellar mass to host cloud mass, is observed to be only a few percents (Myers et al. 1986). Moreover, the free fall time of a molecular cloud (on the order of $1 \mathrm{Myr}$ ) is usually shorter than its age (approximately $2-10 \mathrm{Myr}$ ). These observations imply the existence of mechanisms that prevent the rapid collapse of molecular clouds and explain the low efficiency with which stars are formed.

Different mechanisms have been invoked to regulate star formation. If the potential for magnetic fields embedded in molecular clouds to bring support against self-gravity was recognized early on (Shu. Adams, \& Lizano 1987; Mouschovias \& Ciolek 1999), the role played by interstellar turbulence in the star-forming process was also put forth and is actively studied (Mac Low \& Klessen 2004). The fact that such fundamental questions are still debated owes largely to the lack of observational constraints on existing star formation theories. For example, our knowledge of magnetic field strengths in molecular clouds is mainly based on observations of the Zeeman effect (Crutcher et al. 1993) and the so-called Chandrasekhar-Fermi method (Chandrasekhar \& Fermi 1953), but both types of measurements are difficult to carry out in most parts of a cloud. Similarly, turbulent energy needs to dissipate at small scales to allow for cloud material to collapse and form stars. But the details pertaining to the mechanisms at the origin of turbulence dissipation, whether they are related to viscosities or ambipolar diffusion, are still uncertain.

In this paper, we show how the difference between the turbulent velocity dispersion spectra of coexistent ions and neutrals can shed light on the existence of an ambipolar diffusion range, and be used to estimate magnetic field strengths in molecular clouds. To do so, we first use the method put

forth by Ostriker, Stone, \& Gammie (2001) to study simulations of turbulent molecular clouds to measure the spectral index of the Kolmogorov-type turbulence observed in our velocity dispersion spectra. We then argue that the observed difference between the ion and neutral spectra is due to differences in their respective energy dissipation ranges. Based on the measured spectral index, and the difference in the turbulence spectra, we describe how the strength of magnetic fields can be estimated.

\section{Turbulence in Molecular Clouds}

The observations of molecular line profiles in most parts of giant molecular clouds (GMCs) reveal much wider line widths (on the order of $1 \mathrm{~km} / \mathrm{s}$ and more) than what is expected from thermal motions (a fraction of $1 \mathrm{~km} / \mathrm{s}$ ). While there is no evidence that this motion is due to systematic mass motion, e.g., gravitational collapse, Zuckerman \& Evans (1974) suggested that the large line widths stem from localized supersonic turbulence. This idea is strongly supported by the discovery by Larson (1981) that the line width follows a Kolmogorov-type spectral law, i.e., $\sigma \propto L^{0.38}$, where $\sigma$ stands for the velocity dispersion measured from line profiles and $L$ is the linear size of the observed clouds or cloud cores (see the Fig. 1 of Larson (1981)). Larson's law was derived from the statistics of many clouds/cores of different sizes, but the characteristics of the turbulence velocity spectrum as a function of the length scale vary from cloud to cloud. This can be seen from 
Larson's work, where, for example, data pertaining to the M17 molecular cloud and cores embedded within it imply a smaller spectral index than that (i.e., 0.38) of the overall law mentioned above.

Several methods have been proposed to study turbulence at sub-cloud/core scales (Ossenkopf \& Mac Low 2002). We apply here the technique introduced by Ostriker. Stone, \& Gammie (2001) to our data obtained for M17. Our data consist of the $\operatorname{HCN}(J=4 \rightarrow 3)$ and $\mathrm{HCO}^{+}(J=4 \rightarrow 3)$ rotational transitions observed in an approximately 2 arcmin. $\times 2$ arcmin.-wide region located near the center of M17 with the Caltech Submillimeter Observatory (CSO). These data were previously discussed in details by Houde et al. (2002). Figure 1 shows a log-log plot of the HCN velocity dispersion spectrum as a function of the length scale. The angular resolution of the CSO's 10.4-meter telescope is approximately 20 arcseconds at the frequencies of interest (i.e., approximately $355 \mathrm{GHz}$ and 357 $\mathrm{GHz}$, respectively, for $\mathrm{HCN}$ and $\mathrm{HCO}^{+}$at this transition), and corresponds to the smallest scale on the plot. The data at larger scales were obtained by combining the spectra from adjacent regions to effectively simulate beam sizes of double, triple, and quadruple the original size. In Larson's work it was adequate to approximate the clouds or cores as being spherical in shape, and scales along the line of sight could, therefore, be reasonably estimated from corresponding spatial extents on the sky. On the other hand, at sub-cloud/core scales the sky-projected and line-of-sight scales are no longer directly correlated. Consequently, the scatter at each scale in our data of Figure 1 is mainly due to the range of line-of-sight scales. But, interestingly, Ostriker, Stone, \& Gammie (2001) showed in their simulations of turbulent molecular clouds that the lower envelope of such velocity dispersion distributions traces the actual turbulent velocity spectrum very well.

Two points are worth noting. First, the lower envelope of our HCN data shows a spectral index of 0.18 (as measured from the logarithmic slope), which is smaller than that observed by Larson (1981) for his ensemble of molecular clouds. Second, in contrast to the simulations of Ostriker, Stone, \& Gammie (2001), this spectral index is constant across the dispersion spectrum. The change in spectral index at smaller scales seen in the results of Ostriker, Stone, \& Gammie (2001) (see their Figure 10(a)) is explained by the presence of a turbulent energy dissipation scale. Accordingly, we interpret the constant spectral index of the HCN lower envelope in our data as an indication that we did not reach the dissipation scale in M17 at the spatial resolution with which our data were obtained. That is, our data probe the inertial range.

\section{The Ambipolar Diffusion Scale}

It is usually believed that magnetic fields in the interstellar medium are highly coupled to the gas; this is the so-called "flux freezing" phenomenon. This is one of the most important consequences of the induction equation

$$
\frac{\partial \mathbf{B}}{\partial t}=\nabla \times(\mathbf{v} \times \mathbf{B})+\eta \nabla^{2} \mathbf{B}
$$


where $\mathbf{B}$ is the magnetic field, $\mathbf{v}$ the velocity of the (ionized) gas, and $\eta$ the magnetic diffusivity (assumed to be constant here). The ratio of the convection term to the diffusion term is defined as the magnetic Reynolds number

$$
R_{\mathrm{m}}=\frac{|\nabla \times(\mathbf{v} \times \mathbf{B})|}{\left|\eta \nabla^{2} \mathbf{B}\right|} \sim \frac{V L}{\eta}
$$

where $L$ and $V$ are characteristic spatial and velocity scales, respectively. Under conditions when $R_{\mathrm{m}} \gg 1$, the flux freezing approximation is warranted through Kelvin's vorticity theorem (Choudhuri 1998), as the convection term dominates over the diffusion term in equation (1).

When dealing with weakly ionized plasmas, however, the diffusivity is not limited to that conveyed through $\eta$. The corresponding analysis reveals a more general form for Ohm's law Parker 2007)

$$
\mathbf{E}=\frac{1}{c}\left[-\mathbf{v}_{\mathrm{n}} \times \mathbf{B}+\frac{4 \pi}{c}\left(\eta \mathbf{j}+\alpha \mathbf{j} \times \frac{\mathbf{B}}{B}+\beta \mathbf{j}_{\perp}\right)\right],
$$

where $c$ is the speed of light, $\mathbf{v}_{\mathrm{n}}$ is the velocity of the neutral component of the gas, $\mathbf{j}$ and $\mathbf{j}_{\perp}$ are the current density and its component perpendicular to $\mathbf{B}$, while $\alpha$ and $\beta$ are, respectively, the Hall coefficient and the effective magnetic diffusivity (also known as the Pedersen coefficient (Parker 2007)). It can be shown that for typical conditions encountered in GMCs $\beta \gg \alpha \gg \eta$, and the above relation for the electric field thus simplifies to (Biskamp 2003)

$$
\mathbf{E}=-\frac{1}{c}\left(\mathbf{v}_{\mathrm{n}} \times \mathbf{B}-\frac{4 \pi}{c} \beta \mathbf{j}_{\perp}\right)
$$

The effective magnetic diffusivity is given by

$$
\beta=\frac{B^{2}}{4 \pi n_{\mathrm{i}} \mu \nu_{\mathrm{i}}}
$$

where $n_{\mathrm{i}}$ is the ion density, while $\nu_{\mathrm{i}}$ and $\mu$ are the collision rate of an ion with the neutrals and the mean reduced mass characterizing such collisions, respectively. Although the insertion of equation (4) into Faraday's law of induction does not yield a relation analogous to equation (11) for the induction equation (Brandenburg \& Zweibel 1994), it is nonetheless clear that the flux freezing approximation will cease to be adequate when the effective magnetic diffusivity term dominates over the convective term in equation (4). Consequently, we can define a new effective magnetic Reynolds number with

$$
R_{\mathrm{m}}=\frac{c\left|\mathbf{v}_{\mathrm{n}} \times \mathbf{B}\right|}{4 \pi\left|\beta \mathbf{j}_{\perp}\right|}=\frac{\left|\mathbf{v}_{\mathrm{n}} \times \mathbf{B}\right|}{\left|\beta(\nabla \times \mathbf{B})_{\perp}\right|}
$$




$$
\begin{aligned}
& \sim \frac{V_{\mathrm{n}} L}{\beta} \\
& \sim \frac{4 \pi n_{\mathrm{i}} \mu \nu_{\mathrm{i}} V_{\mathrm{n}} L}{B^{2}},
\end{aligned}
$$

where Ampère's law was substituted for the current density. Based on equation (66), it is clear that although $R_{\mathrm{m}} \gg 1$ for scales usually probed with single-dish observations (i.e., on the order of sizes subtended by the telescope beam), this relation does not necessarily hold true for all the turbulent eddies existing in any given region of a molecular cloud. This is because situations involving small eddies (in the neutral gas) of size $L$ evolving in a region embedded with a (strong) magnetic field will lead to lower effective magnetic Reynolds numbers. In fact, it is expected from the loss in the relative importance of the convective term in equation (4) that the magnetic field, and therefore the ions, will decouple more easily from neutral eddies at such scales, when the effective Reynolds number is on the order of unity or less. This decoupling, or ambipolar diffusion Mestel \& Spitzer 1956), will be responsible for friction forces between the ion and neutral components of the gas, and will thus bring about the dissipation of turbulent energy (Zweibel \& Josafatsson 1983). It is also reasonable to expect that the existence of ambipolar diffusion will be the cause for possible differences between the velocity dispersion spectra of the ions and the neutrals.

We also note that for typical conditions in the interstellar medium (e.g., $B \sim 10 \mu \mathrm{G}, n_{\mathrm{n}} \sim 10^{3}$ $\mathrm{cm}^{-3}$, an ionized fraction of approximately $10^{-6}$, and with $\nu_{\mathrm{i}} \simeq 1.5 \times 10^{-9} n_{\mathrm{n}} \mathrm{s}^{-1}$ for ion/neutral collisions (Nakano 1984)) equation (5) yields an effective magnetic diffusivity $\beta$ of the order of $10^{21} \mathrm{~cm}^{2} \mathrm{~s}^{-1}$, which is much larger than the hydrodynamic viscosity (which is of the order of $10^{16}$ $\mathrm{cm}^{2} \mathrm{~s}^{-1}$ ). The situation also holds for denser parts of molecular clouds such as the ones probed with our data, where both density and magnetic field strength can increase by as much as two or three orders of magnitude. Given this fact, will ambipolar diffusion set an energy dissipation scale in the turbulent energy spectrum that is larger than the scale due to viscosity? This is still an open question, both on observational and theoretical/simulation grounds. For supersonic magnetohydrodynamic turbulence, the fast waves and Alfvén waves are strongly damped at smaller scales when $R_{\mathrm{m}}<1$ (McKee et al. 1993; Balsara 1996). It is, therefore, reasonable to expect steeper energy spectra at ambipolar diffusion scales. But since ions and neutrals are decoupled from each other, their respective energy (and velocity dispersion) spectra must have different slopes at these scales. Which one should be steeper? Here we show that observing the turbulent velocity spectra of coexistent ion and neutral molecular species, even at spatial resolution much lower than ambipolar diffusion scales, can shade some light on these questions.

\section{Comparison of $\mathrm{HCN}$ and $\mathrm{HCO}^{+}$in $\mathrm{M} 17$}

Figure 2 shows in part the same data as in Figure 1, with the exceptions that the vertical axis is now for the square of the velocity dispersion, we used linear scales for both the horizontal and vertical scales, and more importantly data for the other molecular species, $\mathrm{HCO}^{+}$, have been 
added. The use of linear scales for the axes in this figure was chosen to better show the difference between the two data sets as a function of the length scale, as this will become important for the discussion below. As can be seen, the two molecular species show very similar spectral indices for their respective velocity dispersion spectra (i.e., 0.18, as calculated with the lower envelopes), which is expected at this scale because ions are trapped by magnetic fields, which are in turn well-coupled with neutrals due to flux freezing. But note that the lower envelope of $\mathrm{HCO}^{+}$is downshifted from that of HCN. We now turn our attention to identifying the cause for this nearly constant shift of the ion velocity spectrum over all the scales shown in the part of the inertial range probed by our observations.

$\mathrm{HCN}$ and $\mathrm{HCO}^{+}$have been shown to be coexistent in molecular clouds and to have systematic line width differences (Houde et al. 2000a, b, 2002; Lai, Velusamy, \& Langer 2003; Houde et al. 2004). Due in part to its smaller critical density $\mathrm{HCO}^{+}$is generally somewhat more optically thick and will accordingly usually exhibit slightly more extended spatial distributions than HCN (Houde et al. 2000a, b). Both of these properties would suggest that $\mathrm{HCO}^{+}$should have wider line widths than HCN, yet the fact is that its spectral line profiles are consistently narrower in turbulent molecular clouds (such as M17). Houde et al. (2000a) put forth a model, where magnetic fields affect the dynamics of molecular ions through the cyclotron interaction, that can account for the narrowing of their spectral line profiles, which was in turn interpreted as a signature of ambipolar diffusion (Houde et al. 2004).

Here we further argue that the aforementioned observed difference between the two turbulent velocity spectra (as a function of length scale) is also the result of ambipolar diffusion. Even though ambipolar diffusion happens at much smaller scales than that at which our data was obtained, it is still discernible in our observations because the velocity dispersions we measure stem from the contribution of all turbulent eddies with scales approximately equal to, or smaller than, the different beam sizes. To explain the systematically downshifted velocity dispersion spectrum of the ions, it is necessary that

1. Ambipolar diffusion does not set the cut-off range for both the ion and neutral energy spectra.

2. The ion spectral slope must be steeper than that of the neutrals' at ambipolar diffusion scales, as illustrated in Figure 3 (more below).

As mentioned before, a steep drop in the ion velocity dispersion spectrum at ambipolar diffusion scales can be expected due to the fact that most of families of magnetohydrodynamic waves are strongly damped when $R_{\mathrm{m}}<1$. Neutrals, while still partaking in the turbulent energy cascade of the inertial range, should also exhibit a steeper turbulent spectrum than in their inertial range because of friction forces between ions and neutrals. But since the low ionization level will limit the amount of friction, neutrals will still have their energy cut-off set by hydrodynamic viscosity. The square of the observed velocity dispersion at a particular scale is proportional to the integral of the energy spectrum below that scale. Therefore, at all scales larger than those covered by the ambipolar 
diffusion range the difference between the squares of the neutral and ion velocity dispersions will be proportional to the difference of the corresponding integrals between the scale where ambipolar diffusion sets in and the hydrodynamic dissipation scale (see below). This is conveyed through the shaded region in Figure 3 ,

\section{Estimation of the Magnetic Field Strength}

The difference between the ion and neutral velocity dispersion spectra can be used to estimate the strength of the magnetic field. To see how this can be achieved, let us consider the wave number $k^{\prime}=2 \pi / L^{\prime}$ corresponding to turbulent eddies of characteristic size $L^{\prime}$ such that $R_{\mathrm{m}}=V_{\mathrm{n}}^{\prime} L^{\prime} / \beta=1$, with $V_{\mathrm{n}}^{\prime}$ the characteristic velocity of these (neutral) eddies. As discussed earlier, this is the scale at which ambipolar diffusion sets in. For a wave number $K<k^{\prime}$ (i.e., of spatial scale larger than $L^{\prime}$ ), the difference between the squares of the neutral and ion velocity dispersions can be written as

$$
\Delta \sigma_{K}^{2}=\int_{K}^{\infty}\left[F_{\mathrm{n}}(k)-F_{\mathrm{i}}(k)\right] d k
$$

where $F_{\mathrm{n}}(k)$ and $F_{\mathrm{i}}(k)$ are (twice) the turbulent energy spectra density (i.e., per unit mass and wave number) for the neutral and ion species, respectively. But because of the good coupling of the ions and neutral through flux freezing in the inertial range we expect that

$$
F_{\mathrm{n}}(k)=F_{\mathrm{i}}(k), \text { for } k<k^{\prime} .
$$

If we further assume that $F_{\mathrm{i}}(k)$ decreases steeply beyond $k^{\prime}$, then for any wave number $K<k^{\prime}$ the difference of the square of the velocity dispersions (eq. [7) is only a function of $k^{\prime}$ with

$$
\Delta \sigma_{K}^{2} \simeq \Delta \sigma_{k^{\prime}}^{2} \equiv \int_{k^{\prime}}^{\infty} F_{\mathrm{n}}(k) d k, \text { for } K<k^{\prime}
$$

This result is consistent with our observations, since, as was mentioned earlier, our data show such a nearly constant difference between the neutral and ion spectra. More precisely, the measured differences $\Delta \sigma_{K}^{2}$ from the velocity dispersion spectra of Figure 2 are $0.57,0.65,0.57$, and $1.22 \mathrm{~km}^{2} / \mathrm{s}^{2}$ with increasing beam sizes. The discrepancy at the largest spatial scale is easily accounted for by the small amount of statistics available, which prevented us from finding the true lower envelope for both velocity dispersion spectra.

An important consequence that follows from this analysis is that the determination of the decoupling scale $L^{\prime}$ and the neutral velocity dispersion $V_{\mathrm{n}}^{\prime}$ at $L^{\prime}$ would allow for the evaluation of the magnetic field strength through equation (6) by setting $R_{\mathrm{m}}=1$. More precisely, we find that

$$
B^{2}=4 \pi n_{\mathrm{i}} \mu \nu_{\mathrm{i}} V_{\mathrm{n}}^{\prime} L^{\prime}
$$


which can be conveniently transformed using typical parameters for GMCs. For example, if we use mean neutral and ion masses of, respectively, 2.3 and 29 times that of the hydrogen atom, then

$$
B=\left(\frac{L^{\prime}}{0.5 \mathrm{mpc}}\right)^{1 / 2}\left(\frac{V_{\mathrm{n}}^{\prime}}{1 \mathrm{~km} \mathrm{~s}^{-1}}\right)^{1 / 2}\left(\frac{n_{\mathrm{n}}}{10^{6} \mathrm{~cm}^{-3}}\right)\left(\frac{\chi_{\mathrm{i}}}{10^{-7}}\right)^{1 / 2} \mathrm{mG}
$$

where $\chi_{\mathrm{i}}$ is the ionization fraction.

It is important to note that since observations can only reveal the velocity dispersion along the line-of-sight and that the effective magnetic diffusivity term in equation (4) is in a direction perpendicular to the magnetic field, the strength of the field estimated with equations (10) or (11) is only valid for its plane-of-the-sky component.

We now proceed and evaluate $L^{\prime}$ and $V_{\mathrm{n}}^{\prime}$ using our data. First, we note that the velocity dispersion due to an eddy of scale size $k=2 \pi / L$ is given by

$$
V_{\mathrm{n}}^{2}(k)=F_{\mathrm{n}}(k) \Delta k
$$

The energy density $F_{\mathrm{n}}(k)$ can readily be evaluated from our data, since we know that

$$
\begin{aligned}
\sigma_{\mathrm{n}}^{2}(k) & =\int_{k}^{\infty} F_{\mathrm{n}}(\kappa) d \kappa \\
& \simeq b\left(\frac{2 \pi}{k}\right)^{n},
\end{aligned}
$$

where $\sigma_{\mathrm{n}}(k)$ is the neutral velocity dispersion, while $b$ and $n$ are the different fit parameters to the neutral data (see Figure 21). Using the derivative relative to $k$ on this equation and the spectral spread corresponding to a Gaussian beam of full-width-half-magnitude $L$ to estimate $\Delta k$ $(=\sqrt{8 \ln (2)} / L)$, we get from equation (12) that

$$
V_{\mathrm{n}}^{2}(L) \simeq 0.37 b n L^{n}
$$

Moreover, if, as assumed, there is a significant drop of the ion spectrum in its dissipation range (as shown in Figure 3), it then follows that the ion velocity dispersion $\sigma_{\mathrm{i}}\left(k^{\prime}\right)$ observed at $L^{\prime}$ (or $\left.k^{\prime}=2 \pi / L^{\prime}\right)$ will be approximately equal to $V_{\mathrm{n}}^{\prime}$. We, therefore, write

$$
V_{\mathrm{n}}^{\prime 2} \simeq a+b L^{\prime n}
$$

where it should be clear that $-a$ corresponds to $\Delta \sigma_{k^{\prime}}^{2}$, as defined in equation (9) and measured from our data (see Figure 2). Equating equations (14) (at $L^{\prime}$ ) and (15) gives 


$$
L^{\prime n}=\frac{-a}{b(1-0.37 n)}
$$

For M17, the fits to our data yield $a \simeq-0.59 \mathrm{~km}^{2} \mathrm{~s}^{-2}, b \simeq 1.17 \mathrm{~km}^{2} \mathrm{~s}^{-2}$ arcsecond ${ }^{-n}$, and $n \simeq 0.36$. Equation (16) then gives $L^{\prime} \simeq 0.22^{\prime \prime}$ or $1.8 \mathrm{mpc}$ at the distance of M17 (i.e., approximately $1.7 \mathrm{kpc}$ ), and $V_{\mathrm{n}}^{\prime} \simeq 0.30 \mathrm{~km} / \mathrm{s}$.

A precise determination of the magnetic field strength in M17 through equation (11) would require an equally precise knowledge of the gas density $n_{\mathrm{n}}$ and the ionization fraction $\chi_{\mathrm{i}}$, which we do not possess at this time. We can, however, use reasonable values for these parameters and provide an estimate of the magnetic field strength to allow for comparison with previous measurements obtained with other techniques. Therefore, choosing $n_{\mathrm{n}}=10^{6} \mathrm{~cm}^{-3}$, which should be appropriate for $\mathrm{HCN}$ and $\mathrm{HCO}^{+}$at the $J=4 \rightarrow 3$ transition, and $\chi_{\mathrm{i}}=10^{-7}$ for the ionization fraction we calculate a plane-of-the sky magnetic field strength of $B \approx 1.0 \mathrm{mG}$ from equation (11). Although this value compares well with earlier HI and OH Zeeman measurements (Brogan \& Troland 2001) that yielded line-of-sight magnetic field strengths as high as $0.75 \mathrm{mG}$ in this molecular cloud, we should keep in mind that our estimate is probably uncertain to within a factor of a few or even as much as an order of magnitude. We also note that the details of our calculations rest in part on the assumption that the ion velocity dispersion spectrum drops steeply in the ambipolar diffusion range. In the event that this assumption is not realized, the characteristic velocity $V_{\mathrm{n}}^{\prime}$, and the magnetic field strength, would be overestimated through equation (15).

It is important to note that current (e.g., SMA, CARMA) or future (e.g., ALMA) interferometers could provide high enough spatial resolutions to allow for the direct determination of the ambipolar diffusion scale $L^{\prime}$ and the characteristic velocity $V_{\mathrm{n}}^{\prime}$ of an eddy at that scale directly from the velocity dispersion spectra. The determination of $L^{\prime}$ would be straightforward from the velocity dispersion spectrum of the ion, as it would be identified with the scale at which a significant drop in velocity occurs (i.e., the ion cut-off point) or where the neutral and ion spectra depart from one another (see Figure 3).

\section{Summary}

Our analysis of turbulent velocity dispersion spectra from coexistent ion and neutral molecular species can be summarized as follows:

1. We showed that by combining observational spectral line profile data to different scales, in the manner of Ostriker. Stone, \& Gammie (2001), it is possible to probe turbulent velocity dispersion spectra at sub-cloud/core scales.

2. For coexistent ions and neutrals in M17, the turbulent velocity dispersion spectra share the same slope in the inertial range, as expected from the flux freezing approximation, but the 
ion spectrum is downshifted nearly uniformly at all scales relative to that of the neutrals, as shown in Figure 2, This can be explained by the occurrence of ambipolar diffusion at smaller scales where $R_{\mathrm{m}}<1$. The downshifted velocity spectrum of the ions implies that they have a steeper turbulent energy spectrum than the neutrals at ambipolar diffusion scales. It is, therefore, apparent that ambipolar diffusion does not set the energy cut-off range for both ions and neutrals.

3. Since ambipolar diffusion happens in the presence of magnetic fields, the difference between the velocity dispersion spectra of ions and neutrals can be used to estimate magnetic field strengths. For M17, we estimate the ambipolar diffusion scale to approximately $1.8 \mathrm{mpc}$, and the plane-of-the-sky magnetic field strength to approximately $1 \mathrm{mG}$. Because we do not precisely know the gas density and the ionization fraction in M17 this estimate of the magnetic field strength is probably uncertain to within a factor of a few or even as much as an order of magnitude.

We note that using optically thin molecular species, such as the $\mathrm{H}^{13} \mathrm{CN}$ and $\mathrm{H}^{13} \mathrm{CO}^{+}$isotopologues, could be advantageous for this type of study; their systematic line with difference is also reported by Houde et al. (2000b) and Lai, Velusamy, \& Langer (2003). Their advantage would reside in the fact that their corresponding line profiles would not be saturated, unlike the species used in this work in some parts of the molecular cloud under consideration, and the line widths measured would reflect more faithfully the true velocity dispersions of the ion and neutral gas components along the line-of-sight. Moreover, $\mathrm{H}^{13} \mathrm{CN}$ and $\mathrm{H}^{13} \mathrm{CO}^{+}$would allow us to probe magnetic fields at greater depth in star-forming regions, where they are likely to be stronger (Crutcher 1999; Basu 2000).

The authors thank S. Basu, R. H. Hildebrand, E. Keto, P. C. Myers, and E. C. Ostriker for insightful discussions and comments. H. Li's research is funded through a postdoctoral fellowship from the Smithsonian Astrophysical Observatory. M. H.'s research is funded through the NSERC Discovery Grant, Canada Research Chair, Canada Foundation for Innovation, Ontario Innovation Trust, and Western's Academic Development Fund programs. The Caltech Submillimeter Observatory is funded through the NSF grant AST 05-40882 to the California Institute of Technology.

\section{REFERENCES}

Balsara, D. 1996, ApJ, 465, 775

Basu, S. 2000, ApJ, 540, L103

Biskamp, D. 2003, Magnetohydrodynamic Turbulence, (Cambridge: Cambridge University Press)

Brandenburg, A., \& Zweibel, E. G. 1994, ApJ, 427, L91

Brogan, C. L., \& Troland, T. H. 2001, ApJ, 560, 821 
Chandrasekhar, S., \& Fermi, E. 1953, ApJ, 118, 113

Choudhuri, A. R. 1998, The Physics of Fluids and Plasmas: An Introduction for Astrophysicists, (Cambridge: Cambridge University Press)

Crutcher, R. M., Troland, T. H., Goodman, A. A., Heiles, C., Kazès, \& Myers, P. C. 1993, ApJ, 407,175

Crutcher, R. M. 1999, ApJ, 520, 706

Houde, M., Bastien, P., Peng, R., Phillips, T. G., \& Yoshida, H. 2000a, ApJ, 536, 857

Houde, M., Peng, R., Phillips, T. G., Bastien, P., \& Yoshida, H. 2000b, ApJ, 537, 245

Houde, M., Bastien, P., Dotson, J. L., Dowell, C. D., Hildebrand, R. H., Peng, R., Phillips, T. G., Vaillancourt, J. E., \& Yoshida, H. 2002, ApJ, 569, 803

Houde, M., Dowell, C. D., Hildebrand, R. H., Dotson, J. L., Vaillancourt, J. E., Phillips, T. G., Peng, R., \& Bastien, P. 2004, ApJ, 604, 717

Lai, S.-P., Velusamy, T., \& Langer, W. D. 2003, ApJ, 596, L239

Larson, R. B. 1981, MNRAS, 194, 809

McKee, C. F., Zweibel, E. G., Goodman, A. A., \& Heiles, C. 1993, Magnetic fields in star-forming regions - Theory. In Protostars and Planets III, eds. E. H. Levy, J. I. Lunine with the assistance of M. Guerrieri and M.S. Matthews, (Tucson: University of Arizona Press), 327

Mestel, L., \& Spitzer, L., Jr. 1956, MNRAS, 116, 503

Mouschovias, T. C., \& Ciolek, G. E. 1999, in The Origin of Stars ans Planetary Systems, ed. C. J. Lada \& N. D. Kylafis (Kluwer, Dordrecht), 305

Myers, P. C., Dame, T. H., Thaddeus, P., Cohen, R. S., Silverberg, R. F., Dwek, E., \& Hauser, M. G. 1986, ApJ, 301, 398

Mac Low, M.-M., \& Klessen, R. S. 2004, Rev. Mod. Phys., 76, 125

Nakano, T. 1984, Fundam. Cos. Phys., 9, 139

Ossenkopf, V., \& Mac Low, M.-M. 2002, A\&A, 390, 307

Ostriker, E. C., Stone, J. M., \& Gammie, C. F. 2001, ApJ, 546, 980

Parker, E. N. 2007, Conversation on Electric and Magnetic Fields in the Cosmos, (Princeton: Princeton University Press)

Shu, F. H., Adams, F. C., \& Lizano, S. 1987, ARA\&A, 25, 23 
Zuckerman, B., \& Evans, N. J., II 1974, ApJ, 192, 149

Zweibel, E. G., \& Josafatsson, K. 1983, ApJ, 270, 511

This preprint was prepared with the AAS $\mathrm{L}_{\mathrm{E}} \mathrm{X}$ macros v5.2. 


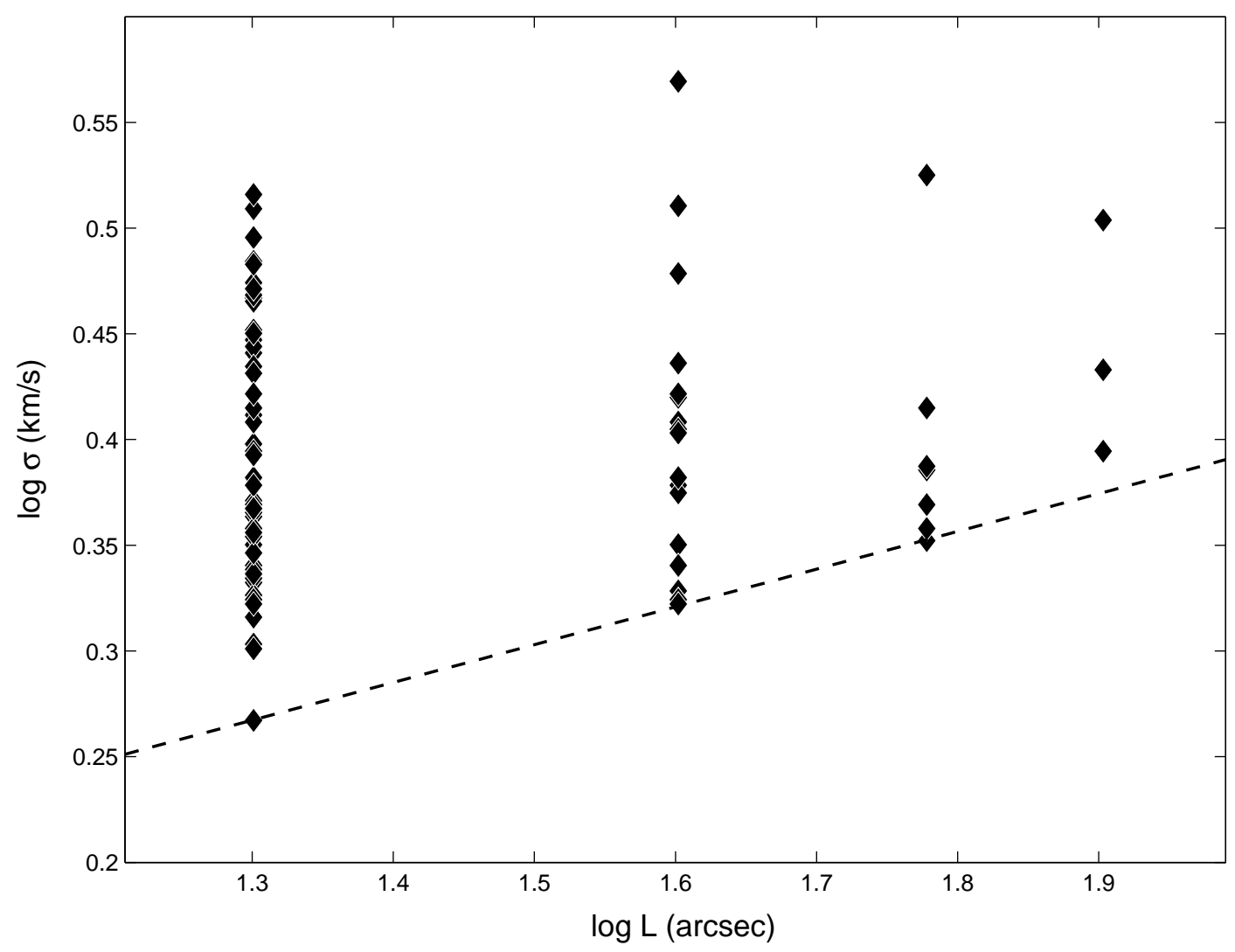

Fig. 1. - The HCN velocity dispersions $\sigma$ obtained from spectral line width measurements at subcloud scales $L$ (using logarithmic scales). The lower envelope can be fitted to a Kolmogorov-type spectrum with a spectral index of approximately 0.18 , as measured from the logarithmic slope. The observed data are at the smallest scale shown (i.e., 20 arcseconds), while adjacent positions were combined to get velocity dispersions at larger scales. 


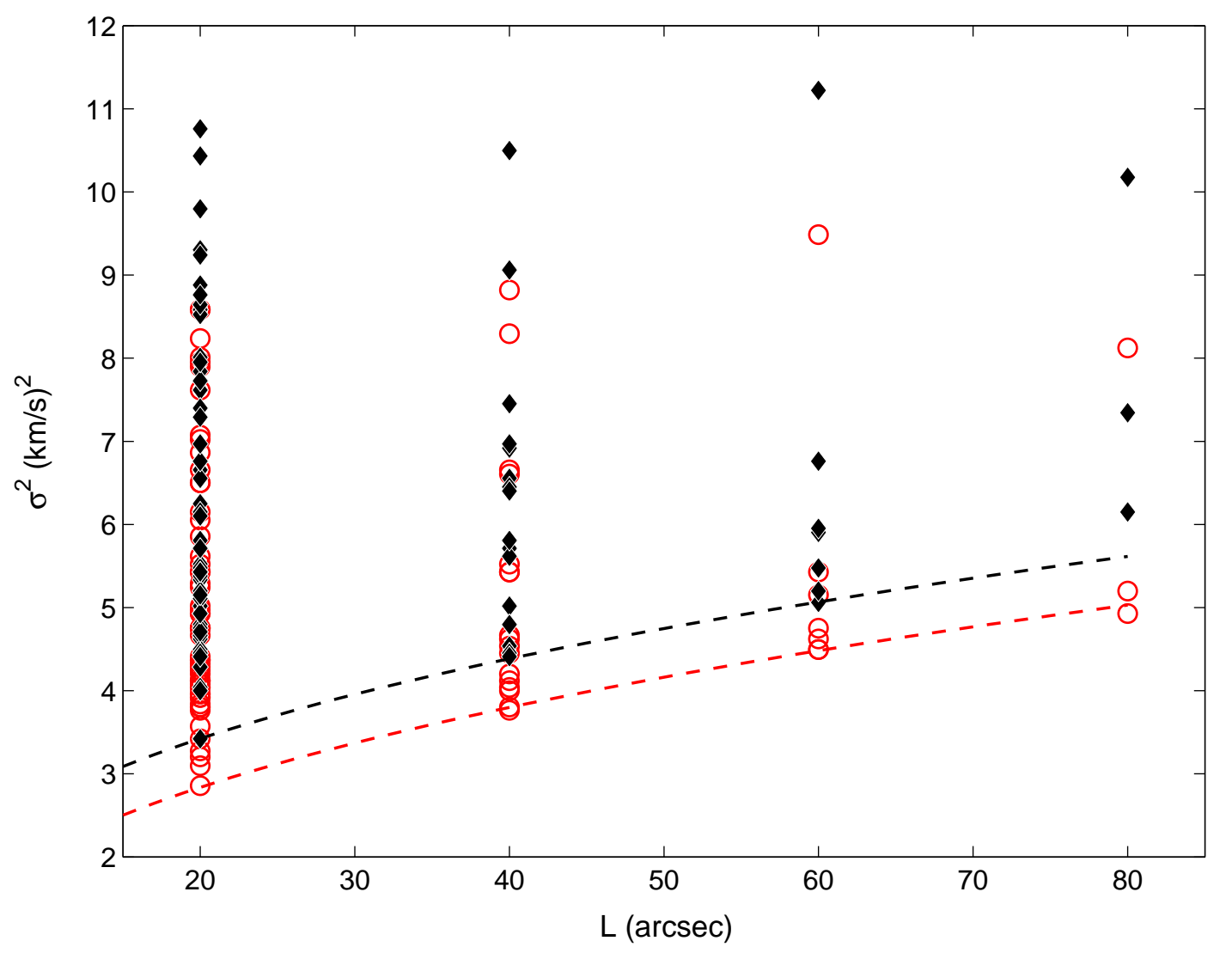

Fig. 2.- Similar to Figure 1, with the exceptions that the vertical axis is now for the square of the velocity dispersion, we used linear scales for both the horizontal and vertical scales, and the data for the other molecular species, $\mathrm{HCO}^{+}$, have been added (in red, $\mathrm{HCN}$ is in black). The $\mathrm{HCN}$ data are fitted for a Kolmogorov-type law $\sigma_{\mathrm{n}}^{2}=b L^{n}$ to their lower envelope. The relative downshift of the $\mathrm{HCO}^{+}$velocity dispersion spectrum is made explicit with the corresponding $\sigma_{\mathrm{i}}^{2}=a+b L^{n}$ fit. The different parameters are $a \simeq-0.59 \mathrm{~km}^{2} \mathrm{~s}^{-2}, b \simeq 1.17 \mathrm{~km}^{2} \mathrm{~s}^{-2}$ arcsecond ${ }^{-n}$, and $n \simeq 0.36$. The measured differences between the two velocity dispersion spectra are $0.57,0.65,0.57$, and 1.22 $\mathrm{km}^{-2} \mathrm{~s}^{-2}$ with increasing beam sizes. The lack of agreement at the largest spatial scale is easily accounted for by the small amount of statistics available, which prevented us from finding the true lower envelope at that scale for both spectra. The uncertainties on the measured velocity dispersions for the lower envelopes are negligible $(<0.01 \mathrm{~km} / \mathrm{s})$ and are thus not shown. 


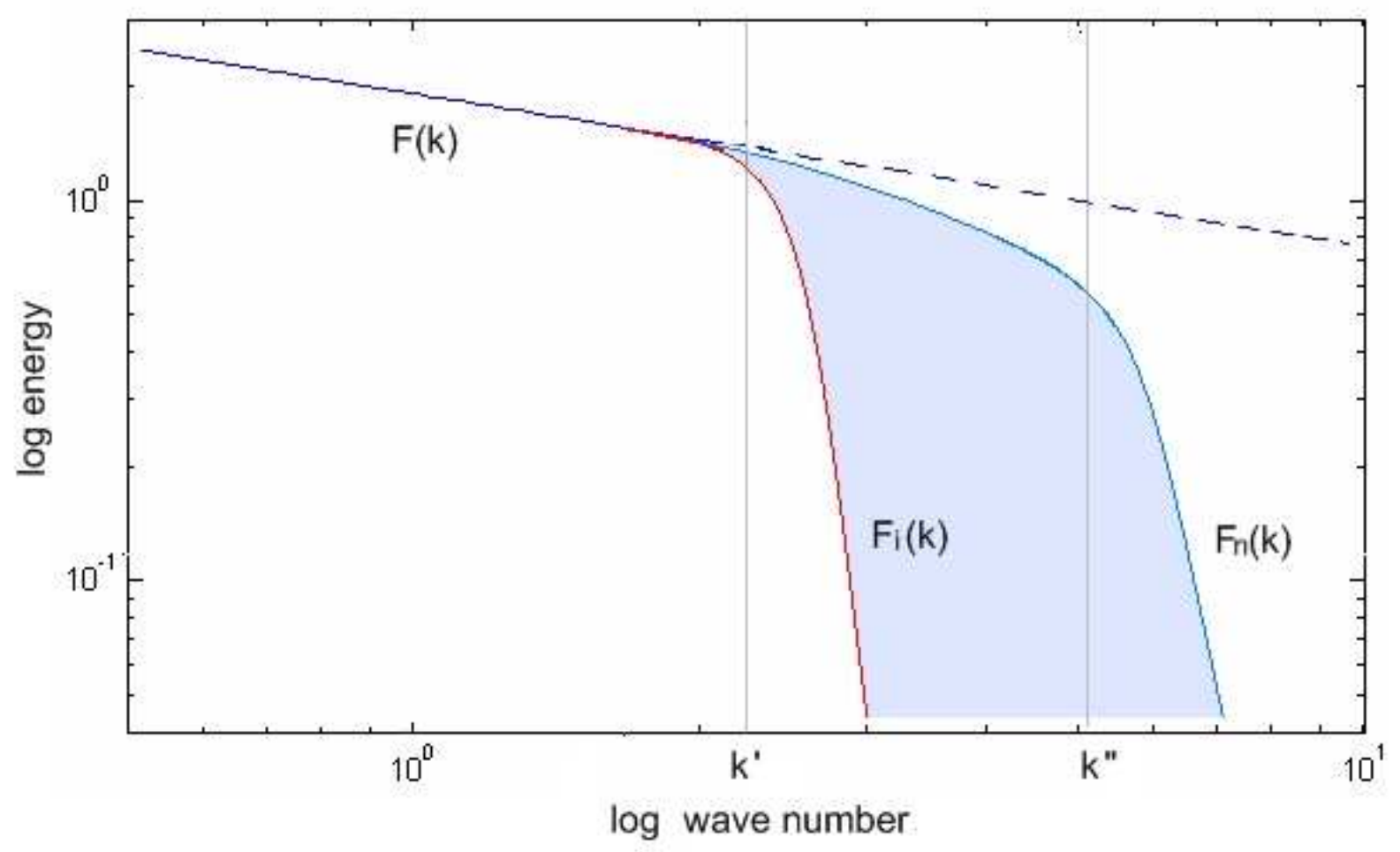

Fig. 3.- An illustration of our explanation for the difference between the ion and neutral velocity spectra of Figure 2 is shown. This is a log-log plot of the turbulent energy versus wave number (inverse of the spatial scale) in arbitrary units. Ambipolar diffusion happens at wave numbers larger than $k^{\prime}$, which is in turn smaller than $k^{\prime \prime}$, where the hydrodynamic viscosity sets in for the neutral spectrum $F_{\mathrm{n}}(k)\left(F_{\mathrm{i}}(k)\right.$ is for the ion spectrum). The energy spectrum in the inertial range, $F(k)$, is common to both species, while ions and neutrals have different spectra at ambipolar diffusion scales. The square of the velocity measured at a particular wave number $K$ is proportional to the integral of the energy spectrum over all wave numbers greater than $K$. The observed difference between the two velocity dispersion spectra is proportional to the shaded area. 